\section{Nomograph for Angular Distributions in Stripping Reactions}

C. R. Lubitz AND W. C. Parkinson

H. M. Randall Laboratory of Physics, Universily of Michigan, Ann Arbor, Michigan

(Received July 21, 1954 ; revised version received January 28,1955 )

1. Introduction. - A rather tedious task in the interpretation of data obtained from stripping reactions is the calculation of the theoretical angular distributions..$^{1,2}$ Normal procedure requires not only that the calculations be performed for various values of orbital angular momenta, but also for a range of values of the "nuclear radius" $r_{0}$. It has been found feasible to reduce these calculations to a nomographic form which reduces considerably the time and labor involved in obtaining the theoretical curves.

The differential cross section for the $(d, p)$ stripping reaction as calculated by Butler is given by Eq. (34) of reference 1 . This was corrected for the finite mass of the target nucleus ${ }^{3}$ by replacing Butler's $Z$ by

$$
Z=\left|\mathbf{k}_{d}-\frac{m_{i}}{m_{f}} \mathbf{k}_{p}\right|^{2} .
$$

For comparison with measured angular distributions, only those parts of Eq. (34) which depend on the angle $\theta_{p}$ are of interest. The equation may be written in the form

$$
\sigma\left(\theta_{p}\right) \sim \Sigma_{\ln } N_{\ln S n i}{ }^{J} F_{\mathbf{l n}^{2}}
$$

where the symbol $\sim$ denotes equality to within a multiplicative factor independent of $\theta_{p}$, and $F_{\ln }$ but not $N_{\ln S_{n i}{ }^{J}}$ is a function of $\theta_{p .}$

The nomograph is designed to give as outputs the (real) values of $F_{l n}$, and $F_{1 n}^{2}$.

2. Description of nomograph.-The primary input quantities are the deuteron energy $E_{d}$, the $Q$ of the reaction, the mass of the target nucleus $m$, the nuclear radius $r_{0}$ and the proton scattering angle $\theta_{p}$, (center-of-mass system). These are converted to the intermediate quantities $Z r_{0}, \kappa_{8} r_{0}$, and $K^{2}$ (as defined in reference 1) by a few simple slide rule calculations together with sheets $A, B$, and $C$ of the nomograph and a table of conversion factors. Sheet $A$, covering the range of $0^{\circ} \leq \theta_{p} \leq 30^{\circ}$, is shown in Fig. 1. Sheets $B$ and $C$ are similar and extend the angle scale $\theta_{p}$ to $180^{\circ}$.

The relative amplitudes $F_{1 \mathrm{n}}$ and cross sections $F_{1 \mathrm{n}}{ }^{2}$ are obtained from the remaining sheets of the nomograph, using $Z r_{0}, \kappa_{8} r_{0}$, and $K^{2}$ as inputs. There are six such sheets, one for each of the values of $\ln =0,1,2,3,4,5$. The sheet for $\ln =1$ is shown in Fig. 2. With the aid of the nomograph a complete angular distribution can be obtained in approximately one half hour.

3. Range and accuracy of nomograph.-The scales have been laid out to cover the following ranges of variables:

$$
\begin{aligned}
8 \leq m & \leq 60 \text { A.M.U. } \\
-\epsilon / 3 & \leq Q / E_{d} \leq 10 / 3 \\
0 & \leq \kappa_{s} r_{0} \leq 6,
\end{aligned}
$$

where $\epsilon$ is the deuteron binding energy in Mev. However, if the variables do not all have the extremes indicated, it is possible for one or more to exceed these limits and still have the results fall within the nomograph scales. The requirement that $x_{s}$ be real requires that $Q \geq-\epsilon=-2.226 \mathrm{Mev}$. A different nomographic layout is necessary for imaginary values of $\kappa_{s}$, corresponding to free neutrons.

The scale markers on the nomograph are so placed that the major inaccuracy in the result occurs due to inexact positioning and reading of the connecting lines. An accuracy of a few percent can be obtained without difficulty. In particular the positions of the peaks and zeros of the curve are usually within one or two degrees of the values obtained by numerical calculation. When the values of the input quantities fall outside the range of the nomograph scales, the formulas included with the nomograph may be used to perform a numerical calculation.

The complete nomograph consists of: (a) nine nomographic sheets (as described in the foregoing), (b) a table of conversion factors and a sample work sheet for the slide rule calculations, and (c) a set of instructions for the use of the nomograph, including a description of the changes made in the form of Butler's Eq. (34)
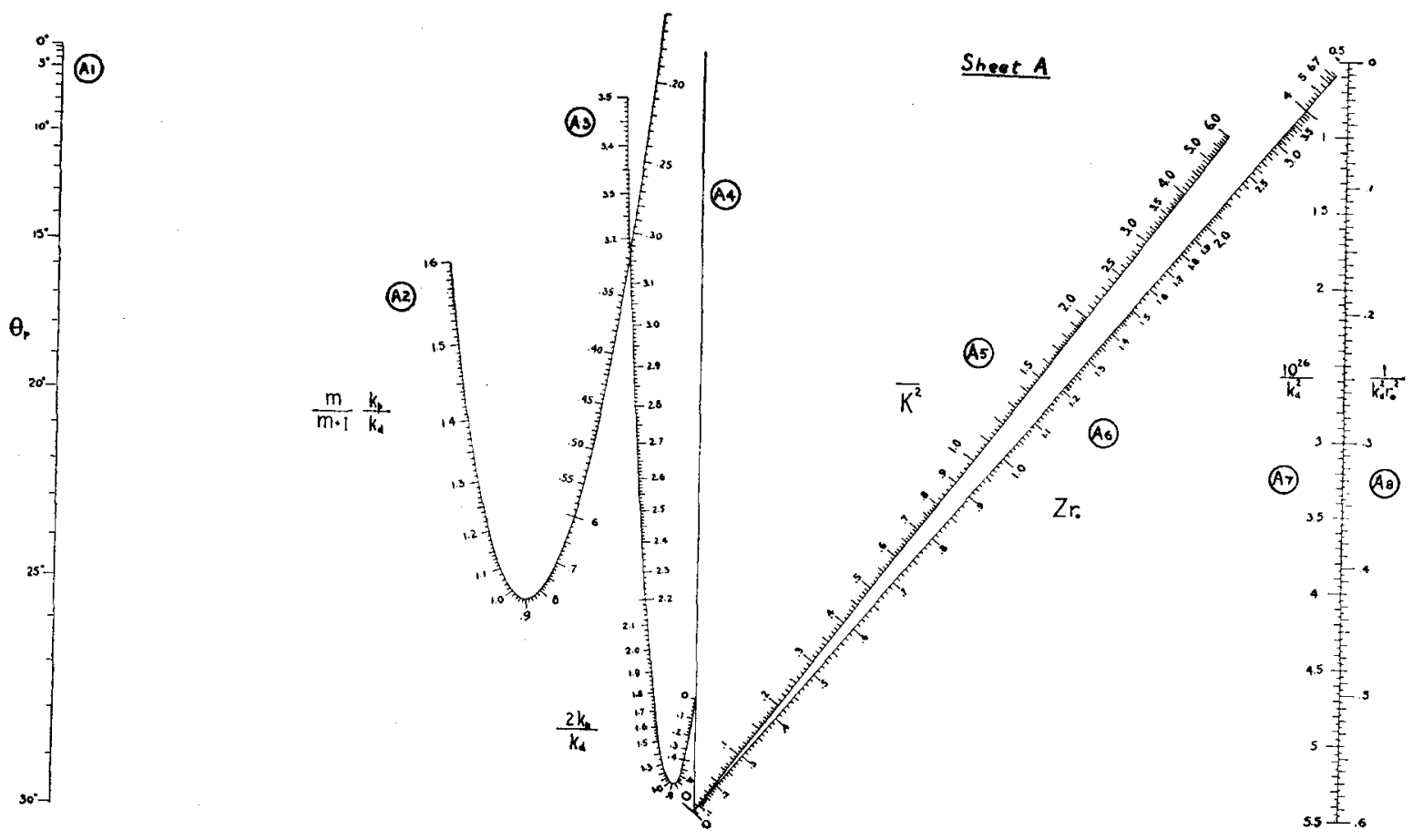

Fig. 1. 

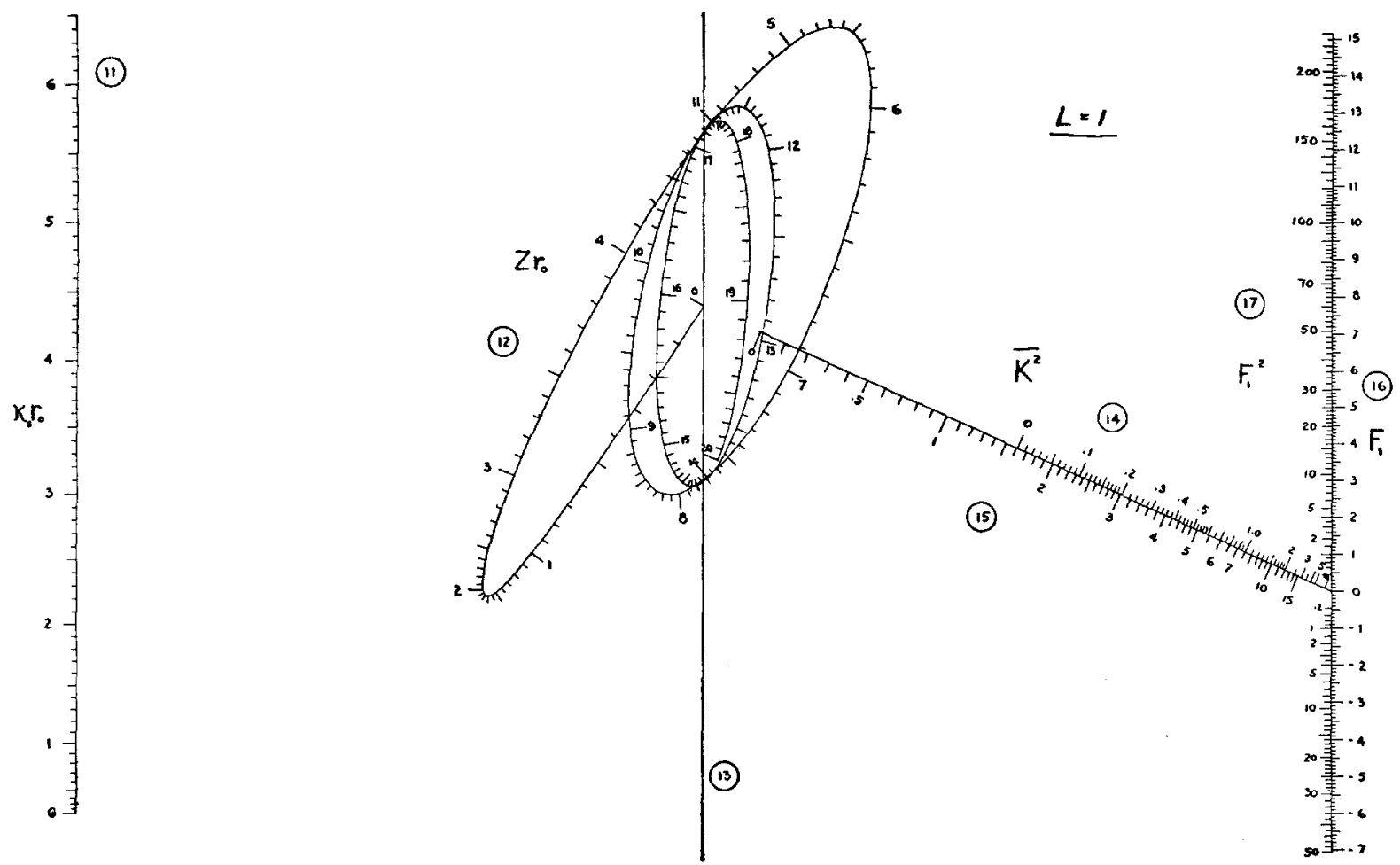

FIG. 2.

to allow a nomographic presentation and a complete listing of all equations solved by the nomograph. it.

The nomograph will be made available at cost to those desiring

1S. T. Butler, Proc. Roy. Soc. (London) 208, 559 (1951)

, Bhatia. Huang, Huby, and Newns. Phil. Mag. 43,485 (1952)

B. T. Butler and E. E. Salpeter, Phys, Rev. 88, 133 (1952).

\section{Laboratory and Shop Notes}

Brief contributions in any field of instrumentation or technique within the field of the Journal can be accorded earlier publication if submitted for this section. Contributions should in generaly not exceed 500 roords.

\section{Method of Reducing Zero Error and Drift in Breaker Type dc Amplifiers}

T. M. DAuphinee

Division of Physics, National Research Council, Ottawa, Canada

(Received Februrary 9, 1955)

$\mathbf{B}^{\mathrm{R}}$

REAKER type dc amplifiers operating to dc input levels of $0.1 \mu \mathrm{v}$ or less require very careful control of intrusive thermal and contact voltages ${ }^{1}$ in the breakers and the primary winding of the input transformer [e.g. Fig. 1(a)]. The breaker action produces a series of current pulses from such a voltage, with the result that a spurious signal is fed into the amplifier which may cause either zero error or drift. Since the transformer normally has a very high impedance secondary, it is desirable to have it located as close to the first amplifier tube as possible and thermal shielding is often difficult, frequently necessitating transformers of very special design.

First-order cancellation of thermal emf's in the input transformer may be obtained as shown in Fig. 1(b) by using a doublepole, double-throw input chopper to give complete reversal of the primary winding with respect to the signal [as opposed to a single-pole chopper which switches from one end to the other of a center-tapped winding, Fig. 1(a)]. This cancellation holds only to the extent that the phasing and effective sensitivity of the two halves of the chopper cycle can be made perfectly symmetric; the error caused by a difference $\Delta t$ between the times of connection $(t)$ in the two positions being approximately $e_{r} \Delta t / 2 t$ where $e_{r}$ is the thermal emf in the transformer.

However, the complete cancellation of transformer emf's can be effected by the introduction of a high-quality condenser $C$ [Fig. 1 (c)] of suitable value in series with the transformer winding. Because of the reversal of the primary winding, any dc signal voltage arising at the input to the choppers will give rise on the output side to a square wave ac which will pass the condenser. On the other hand, a dc voltage arising in the transformer or the leads to the chopper will result in a unidirectional current in the primary winding, which can persist only until a cancelling voltage has been built up in the condenser, the combination of condenser and transformer thereafter behaving as if no thermal voltage existed. We can, therefore, completely ignore all thermal emf's on the transformer side of the chopper, and the transformer itself can be mounted beside the first amplifier tube without any kind of thermal shielding.

With one amplifier used in this laboratory, ${ }^{2}$ the addition of the condenser reduced the zero error from approximately $0.5 \mu \mathrm{v}$, the precise value being strongly dependent on chopper setting, to less than $0.01 \mu \mathrm{v}$ with negligible dependence on chopper setting.

For symmetry purposes it is preferable to use a two-section primary with the condenser between the two sections as shown. The condenser need not cause an appreciable drop in sensitivity 